\title{
Integración económica y desequilibrios territoriales en la Unión Europea
}

\begin{abstract}
Given the lack of theoretical consensus about the spatial effects of economic integration processes, this paper studies the impact of Eumpean economic integration on regional disparities from an empirical perspective. The analysis highlights that different stages of integnation are associated with convergence among European states in inflation and unemployment rates and in economic cyles, as well as with convergence in GDP per capita. However, when the analysis is performed taking into account the distortions linked to the state effect, convergence gives ways in numerous cases, to divergence. From this point of view, European integration is fostering the economic dynamism of core areas, whereas many peripheral regions are encountering increasing difficulties to compete in integrated markets.
\end{abstract}

Keywords: convergence, divergence, European integration, regional disparities, EU.

\section{Resumen}

\begin{abstract}
Ante la falta de acuerdo de las teorías económicas sobre los efectos territoriales de los procesos de integración económica, este artículo analiza desde una base empírica el impacto de la integración económica europea sobre los desequilibrios regionales. El estudio pone de manifiesto que, a medida que se avanza en el proceso de integración, se produce una convergencia entre estados en los ámbitos de inflación, desempleo y ciclo económico, así como en el PIB per cápita. Sin embargo, cuando el análisis se realiza teniendo en cuenta las distorsiones provocadas por el efecto Estado, la convergencia desaparece y da paso, en numerosos casos, a divergencia. Desde este punto de vista, la integración eur opea está favoreciendo el dinamismo económico de los espacios centrales, mientras que numerosas regiones periféricas tienen cada vez mayores dificultades para competir en un mercado cada vez más integrado.
\end{abstract}

Palabras clave: convergencia, divergencia, integración europea, desequilibrios regionales, UE. 


\section{Introducción}

$\mathrm{E}$ n las dos últimas décadas se han producido importantes progresos en el proceso de integración europea. Mientras que entre los años '50 y los '80 los países de Europea Occidental habían limitado sus ansias integradoras en el ámbito económico a la existencia de una unión aduanera, los '90 se han caracterizado por una marcada aceleración del proceso integrador: la formación del Mercado Único el 1 de enero de 1993, seguida por la puesta en marcha del la Unión Económica y Monetaria el 1 de enero de 1999 (Rodríguez-Pose, 2001).

Este proceso de integración acelerada está teniendo un profundo efecto sobre la economía de todos y cada uno de los estados miembros de la Unión Europea (UE). El resultado fundamental está siendo una convergencia de las pautas y ciclos económicos de la práctica totalidad de los estados que forman parte de la Unión Monetaria. Desde un punto de vista territorial, en cambio, las consecuencias son todavía inciertas (Barry, 2003; Begg, 2003). ¿Se están beneficiando todos los países y regiones europeas por igual del proceso de integración económica? ¿ O, por el contrario, la integración económica está fav oreciendo a ciertos espacios por encima de otros? En el caso de que la respuesta a esta última pregunta sea afirmativa, se abren nuevos interrogantes. ¿Guáles son los espacios que están siendo favorecidos por el proceso? ¿Y el resultado de este proceso es una mayor convergencia o divergencia territorial? No existe una teoría dominante que dé una respuesta única a estas cuestiones. Más bien al contrario, las teorías económicas defienden posturas diversas, cuando no abiertamente enfrentadas (Puga, 2002; Magrini, 2003). Por ello, la respuesta a estas cuestiones debe venir fundamentalmente del estudio de la evolu-

\footnotetext{
Profesor de Geografía Humana, London School of Economics. E-mail: A.Rodriguez-Pose@lse.ac.uk

"* P rofesor de Análisis E conómico Espacial, Universidad de Tesalia. E-mail: petrakos@uth.gr

${ }^{* *}$ Versión reformada y ampliada de un trabajo presentado a la XXVII Reunión de Estudios Regionales. Los autores agradecen los comentarios de los árbitros y la financiación del Royal-S ociety Wolfson Research Merit Award y de Philip Leverhulme Prize.
}

ción reciente de las disparidades económicas y de cómo éstas han sido afectadas por los distintos estadios de integración.

Este es el objetivo principal de este estudio: intentar aportar cierta luz sobre el impacto que el proceso de integración económica, y en especial el Mercado Único y la Unión Monetaria, está teniendo sobre los desequilibrios económicos a nivel nacional y regional en la UE. Para alcanzar este objetivo el estudio se estr uctura en cinco apartados. Los dos primeros repasan brevemente los diferentes estadios del proceso de integración europea y las distintas teorías sobre convergencia y divergencia económica. El tercero estudia el impacto de la integración sobre las economías europeas, prestando especial atención a la relación entre ciclos económicos nacionales y ciclo económico europeo. En la cuarta parte se analiza la evolución de las disparidades regionales en el interior de los países miembros de la UE en los distintos estadios de integración económica. Finalmente, en la quinta y última parte se presentan las conclusiones y el posible alcance político de los resultados.

\section{La aceleración del proceso de integración económico}

En los 30 años que siguieron a la firma del Tratado de Roma en 1957, las Comunidades Europeas (CCEE), a pesar de ser popularmente conocidas bajo el nombre de Mercado Común, distaban mucho de ser un verdadero mercado común. La libre circulación de los factores económicos estaba seriamente restringida. No existía la libre movilidad de personas ni de servicios y, aunque se habían realizado grandes progresos en la circulación de capitales, esto había sido más consecuencia del inicio del proceso de globalización que del proceso de integración político-económica. De hecho, la integración económica durante este periodo se había ceñido a permitir desde finales de los años '60 -y de manera harto imperfecta- la libre circulación de bienes entre los Estados Miembros, y a adoptar una serie de tarifas y barreras arancelarias comunes. En definitiva, las CCEE, hasta mediados de los años '80, no constituían más que una forma imperfecta de unión aduanera (Cecchini, 1988). 
La mayoría de los Estados Miembros se encontraban cómodos en esta situación. La libre circulación de bienes favorecía el comercio en el interior de Europa, y este nivel de integración prácticamente no alteraba la capacidad de maniobra económica de todos y cada uno de los gobiernos nacionales. Las principales armas de política económica (la política fiscal y la política monetaria) se encontraban firmemente en manos de los ministros de economía nacionales, y la facultad de las CCEE para intervenir en estos asuntos era sumamente limitada, cuando no inexistente.

Durante gran parte de las tres décadas que siguieron a la formación de las CCEE las perspectivas de cambio en el statu quo eran remotas. Los Estados Miembros veían con recelo una mayor integración económica, y la evidencia histórica iba en contra de cualquier avance en esta dirección. Ningún experimento de integración llevado a cabo en la era moderna había conseguido sobrepasar hasta entonces, utilizando métodos pacíficos, el estadio de Unión Aduanera (Puga, 2002; Tondl, 2002).

Sin embargo, el statu quo se ha transformado de manera radical en los últimos años. En apenas una década y media, Europa ha quemado etapas de integración económica a mucha mayor velocidad que en el pasado, y con mayor rapidez que en cualquier otro proceso histórico de integración por vías pacíficas (Rodríguez-Pose, 2001). Primero se pasó de la Unión Aduanera al Mercado Único. En 1986, con la firma del Acta Única, los miembros de las CCEE decidieron la entrada en vigor del Mercado Único el 1 de enero de 1993, en el que se permite no sólo la libre circulación de bienes, sino también de personas, capitales y servicios. Al mismo tiempo que se ultimaban los preparativos del Mercado Único, los países miembros ya estaban preparándose para la siguiente etapa de integración. El Tratado de Maastricht estableció las bases para la Unión Monetaria, que culminaría con la adopción del Euro en once Estados miembros ${ }^{1}$ de la Unión Europea el 1 de enero de 1999.

¿Cuáles son los factores que hacen que una agrupación de estados soberanos, constituida du- rante la mayor parte de su existencia como una Unión Aduanera, decida -en un periodo de tiempo relativamente corto- embarcarse en un proceso de integración económica mucho más profundo? Las razones detrás del rápido progreso en el proceso de integración económica son múltiples y de diversa índole. Los motivos de carácter económico se solapan con los de índole política e incluso social. Pero quizás la causa principal de la aceleración en el proceso de integración económica ha sido la necesidad europea de responder al reto de la globalización económica y conseguir mantener sus niveles de competitividad (Cappelen et al., 2003). La percepción de que una Europa dividida en mercados nacionales era menos competitiva que Estados Unidos y Japón, en un mundo cada vez más integrado, pesó en gran medida en la aceleración del proceso integrador (Cecchini, 1988). La percepción dominante era que la creación del Mercado Único y la Unión Monetaria no sólo traerían mayores posibilidades de obtener economías de escala y economías de aglomeración, sino que impulsarían los avances tecnológicos y la innovación y fomentarían la reestructuración de sectores poco competitivos (Barry, 2003). La integración de los mercados europeos traería también ahorro y mayor eficacia al acabar con las políticas de campeones nacionales, que a menudo habían resultado en una menor competitividad de las empresas europeas en sectores clave en los mercados internacionales (Cecchini, 1988).

Si los argumentos a favor de la integración de los mercados europeos en un mundo en globalización eran poderosos y se generó un consenso en torno a ellos que llevó a la acción políti$\mathrm{ca}$, las opiniones sobre el posible impacto territorial del proceso de integración económica no eran -ni son- tan unánimes (Cuadrado-Roura, 2001; Begg, 2003). Las teorías económicas al respecto no proporcionan una única respuesta. En el siguiente apartado se repasan de manera breve las diferentes teorías que analizan el impacto territorial de la integración económica y sus posibles implicaciones políticas.

${ }^{1}$ Doce tras la incorporación de Grecia el 1 de enero de 2001. 


\section{Integración económica y teorías de convergencia y divergencia}

El proceso de integración económica europea ha estado acompañado por un renacimiento del interés en las teorías de crecimiento económico. El debate sobre convergencia económica ha pasado a ocupar un lugar estelar en este renacimiento (Magrini, 2003). Durante la última década y media se ha escrito mucho sobre la evolución económica de estados y regiones, y sobre el posible impacto territorial de la globalización y de los procesos de integración. Si atendemos al resultado de estos procesos, podemos distinguir entre dos grupos de teorías: aquellas que afirman que cada vez se está produciendo una mayor convergencia económica entre espacios, frente a las que defienden que la combinación de distintos factores está fomentando una cada vez mayor concentración de la actividad económica en determinados lugares, y por consiguiente, la divergencia.

Entre las primeras cabe reseñar las teorías del comercio de carácter neo-ricardiano. Desde este punto de vista, la integración económica estaría dando lugar a un movimiento cruzado de factores que a la larga llevaría a un estado de equilibrio y a una reducción de las disparidades económicas entre estados. La apertura de fronteras, la liberalización del comercio y la libre movilidad de los factores económicos producirían un flujo cruzado de capital y tecnología, de un lado, y mano de obra, del otro. El capital y la tecnología fluirían desde aquellas regiones donde los costes laborales y de producción son más altos hacia los espacios con costes más bajos. La mano de obra emigraría en sentido opuesto en busca de salarios más altos. Los flujos contrapuestos de inversión y tecnología desde el centro a la periferia, y de capital humano desde la periferia al centro, contribuirían a generar un may or equilibrio entre territorios, y por tanto, convergencia. Desde esta perspectiva, la integración económica traería como resultado una reducción de las disparidades económicas en la Unión Europea, obviando así la necesidad de poner en marcha políticas de desarrollo económico y de cohesión.

La teoría neoclásica es aun más radical en este aspecto que las teorías de corte neo-Ricardiano
(Barro \& Sala-i-Martín, 1991). El enfoque neoclásico percibe la convergencia económica como un factor casi automático e independiente del grado de integración económica. De acuerdo con los postulados neoclásicos de competencia perfecta y tecnología exógena, el crecimiento económico se produce fundamentalmente mediante la aportación de capital, a través de la inversión y el ahorro. La dependencia del crecimiento del factor capital genera rendimientos de escala constantes o decrecientes, ya que los efectos positivos del ahorro y de la inversión decrecen a medida que aumenta la proporción entre el capital y la mano de obra (Pack, 1994). Y las consecuencias de la existencia de rendimientos decrecientes es la convergencia entre territorios, independientemente del grado de integración económica de los mismos. Desde el punto de vista neoclásico, las políticas de desarrollo tampoco son necesarias, ya que los desequilibrios económicos tenderán a corregirse de manera automática sin necesidad de intervención (Puga, 1999).

Frente a las teorías que defienden la convergencia y la reducción de las disparidades territoriales -como consecuencia o no de un proceso de integración económica-, otras teorías apoyan la idea de que los procesos de integración económica pueden generar divergencia y mayores desequilibrios entre espacios. Para las teorías de crecimiento endógeno, desarrolladas por Romer (1986) y Lucas (1988), la inclusión de la tecnología y del capital humano como variables endógenas en los modelos de crecimiento produce un importante cambio con respecto a las teorías neoclásicas. La posesión de alta tecnología o de una mayor capacidad de innovación permite a los espacios que gozan de estas ventajas obtener mayor es rendimientos que aquellos que parten de una base tecnológica más baja, o que carecen de la capacidad o de las estructuras adecuadas para asimilar la innovación (Rodríguez-Pose, 2001). Esto hace que, frente al postulado neoclásico de rendimientos constantes o decrecientes que generaban convergencia económica, se puedan producir rendimientos crecientes de escala que dan lugar a procesos de divergencia.

Los procesos de divergencia previstos por la teoría del crecimiento endógeno se ven aumenta- 
dos, de acuerdo con la teoría neo-schumpeteriana de la innovación, por los costes ligados a la difusión del conocimiento tecnológico (Dosi, 1988; Audretsch \& Feldman, 1996). Al ser la tecnología un bien de carácter semipúblico o parcialmente público, la apropiación de los beneficios derivados de una mayor capacidad tecnológica resulta difícil (Storper, 1997). Esto da lugar a que se produzcan transferencias de conocimiento tecnológico (lo que se conoce en inglés como spillovers de conocimiento) que benefician a empresas y/o territorios vecinos a aquellos en donde se ha producido la innovación. Sin embargo, la transmisión de los spillovers del conocimiento no se produce de manera equitativa en el espacio y su coste aumenta con la distancia (Audretsch \& Feldman, 1996). Por consiguiente, los spillovers tienden a generar efectos multiplicador es muy importantes precisamente en aquellos espacios donde hay ya una alta concentración de tecnología y actividad innovativa, que suelen coincidir con los espacios centrales (Archibugi \& Iammarino, 2002). Se crean pues en estas zonas círculos virtuosos del conocimiento que contribuyen a una más rápida difusión y asimilación de la innovación en las áreas centrales. Los espacios periféricos, por el contrario, no sólo se encuentran más alejados para beneficiarse de la difusión del conocimiento tecnológico, sino que a menudo también su estructura industrial y empresarial no es la más adecuada para la recepción y asimilación de la innovación (Cohen \& Levinthal, 1989). La existencia de empresas aisladas -con frecuencia de menor tamaño y poco competitivas-, de una mano de obra menos cualificada y el aislamiento geográfico no sólo no favorecen la innovación, sino que a menudo fomentan el rechazo o la asimilación parcial de la innovación procedente del exterior (Rodríguez-Pose, 2001). Los procesos de integración económica benefician pues a las ár eas centrales, ya que generan efectos de reestructuración económica y de economías de escala y de aglomeración que dan lugar a una cada vez mayor concentración de la investigación y de la tecnología en aquellas áreas bien dotadas de tecnología y capital humano, frente a zonas donde, con frecuencia, el desarrollo tecnológico se había mantenido gracias a la protección de mercados o a la inversión por parte del sector público (Rodríguez-Pose, 2001).
Las diferentes dotaciones y comportamientos del centro y de las periferias en el campo tecnológico dan como resultado un proceso de concentración de la actividad económica en los centros, mientras que las periferias tienden a perder recursos económicos, provocando un incremento de las disparidades entre unos centros cada vez más prósperos y unas periferias cada vez más dependientes.

Esta visión de mayor concentración de la actividad económica y de la posibilidad de procesos de divergencia, es corroborada en parte por la llamada "nueva geografía económica". Según Fujita, Krugman y Venables (1999), la combinación de factor es como una mayor accesibilidad y una mejor dotación de recursos humanos con la existencia de fuertes vínculos empresariales y de economías de escala y aglomeración en los centros, y menores costes de transporte, favorece una cada vez mayor concentración de la actividad económica. Los procesos de integración económica en Europa contribuirían al movimiento de recursos desde la periferia al centro, ya que aumentaría la importancia de factores como la cercanía al mercado, de los vínculos entre empresas y de las economías de aglomeración. El resultado sería de nuevo una cada vez mayor concentración de la actividad económica y mayor divergencia (Ottaviano \& Puga, 1998; Hanson, 1998).

\section{Integración económica europea y convergencia económica nacional}

Como hemos visto, las teorías económicas no proporcionan una respuesta unívoca sobre el posible impacto territorial del proceso de integración económica en Europea Occidental. Las posibles consecuencias territoriales de los procesos de integración económica varían según el enfoque teórico que se adopte.

A la vista del desacuerdo entre los distintos enfoques teóricos se hace necesario r ecurrir al análisis empírico para establecer cuáles son las principales transformaciones económicas ligadas al proceso de integración europea, y si la tendencia que predomina es a la convergencia o divergencia. En este apartado trataremos las transformaciones a 
nivel nacional, mientras que en el siguiente apartado analizaremos los cambios en las pautas de crecimiento regional en el interior de cada uno de los estados miembros de la UE para los que se dispone de series completas de datos.

\subsection{Integración económica y distintas convergencias}

La aceleración del proceso de integración económica en Europa ha traído como consecuencia una convergencia entre las distintas economías nacionales en diversos aspectos. El primer área de convergencia ha sido la convergencia nominal (Cuadrado Roura, 1996; De la Dehesa, 1998). Las reformas ligadas al Mercado Único y el cumplimiento de las condiciones establecidas en el Tratado de Maastricht para formar parte de la Unión Monetaria ha hecho que las economías europeas tengan hoy comportamientos mucho más semejantes que hace un par de décadas.

La convergencia nominal se ha producido en diversos ámbitos. Quizás la medida más importante ha sido la adopción del Euro como moneda única. La desaparición de las distintas monedas nacionales a favor de una moneda común no sólo representa una importante cesión de soberanía económica por parte de los estados miembros y un gesto político de gran trascendencia, sino que también se antoja como un paso fundamental para la total unión económica. El establecimiento del Banco Central Europeo (BCE) ha contribuido a que los estados cedan también su soberanía en materia de política monetaria. Así, frente a las grandes difer encias en los tipos de interés que se apreciaban hace tan sólo una década entre los estados de la UE, hoy en día en la zona Euro existe un solo tipo de interés fijado por el BCE.

El cumplimiento de los criterios fijados en el Tratado de Maastricht para entrar en la moneda única ha llevado a una cada vez mayor convergencia en otros campos (Begg, 2003). Quizás el campo donde se ha producido un acercamiento mayor ha sido en el de la inflación. Hasta principios de la década de los '90 las diferencias de inflación entre países que ahora son miembros de la Unión Monetaria eran notables. En 1990 la tasa de inflación en Grecia se situaba en torno al 20\%, y en
Portugal alrededor del 13\%, mientras que en Alemania o los Países Bajos apenas superaba el 2\% (Gráfico 1). Desde la firma del Tratado de Maastricht se ha producido una convergencia constante en las tasas de inflación, que llegó a su cúspide en 1998 (Gráfico 1). Aunque desde la adopción de la moneda única se ha producido una ligera divergencia en las tasas de inflación entre algunos países con relativamente alta y baja inflación (Irlanda, Países Bajos y España, y Francia y Suecia, respectivamente), las diferencias son del orden de 1 a 3, frente al 1 a 10 de principios de los '90. Desde la firma del Tratado de Maastricht se ha producido también una reducción importante de las disparidades entre estados en términos de déficit público, y en menor medida, en términos de deuda pública.

Otro resultado ligado en parte al proceso de integración ha sido la fuerte convergencia entre países en niveles de desempleo (Demertzis \& Hughes Hallet, 1996; Martin, 1997; Overman \& Puga, 2003). Hasta más allá de mediados de los años '90 en la UE convivían estados con tasas de desempleo superiores o cercanas a niveles del $20 \%$ de la población activa (España e Irlanda y Finlandia, respectivamente), con países como Luxemburgo o los Países Bajos, donde la tasa de desempleo se situaba por debajo del $5 \%$. El proceso de integración europea y la aplicación de medidas destinadas a reformar y flexibilizar el mercado laboral han hecho que esas diferencias se reduzcan en los últimos años. Si bien el nivel de convergencia en tasas de desempleo ha sido mucho menos espectacular que en los niveles de inflación, en 2002 el nivel de desempleo en el país con la tasa más alta (España) apenas supera a la media de la zona Euro en un 50\%, cuando en 1995 se acercaba al 250\%.

Pero quizás el ámbito en el que el proceso de integración económica europea se haya dejado sentir más es en el de la convergencia en los ciclos económicos (Hess \& Shin, 1997). Cada estadio del proceso de integración europea ha traído consigo un acercamiento del ciclo económico de la mayoría de los países de la UE al ciclo europeo (Petrakos, Rodríguez-Pose y Rovolis, 2003). Las etapas de integración han tendido pues a homogeneizar el comportamiento de las pautas de crecimiento y de la economía entre países. 
Gráfico 1. Convergencia nacional en las tasas de inflación en los países de la zona Euro.

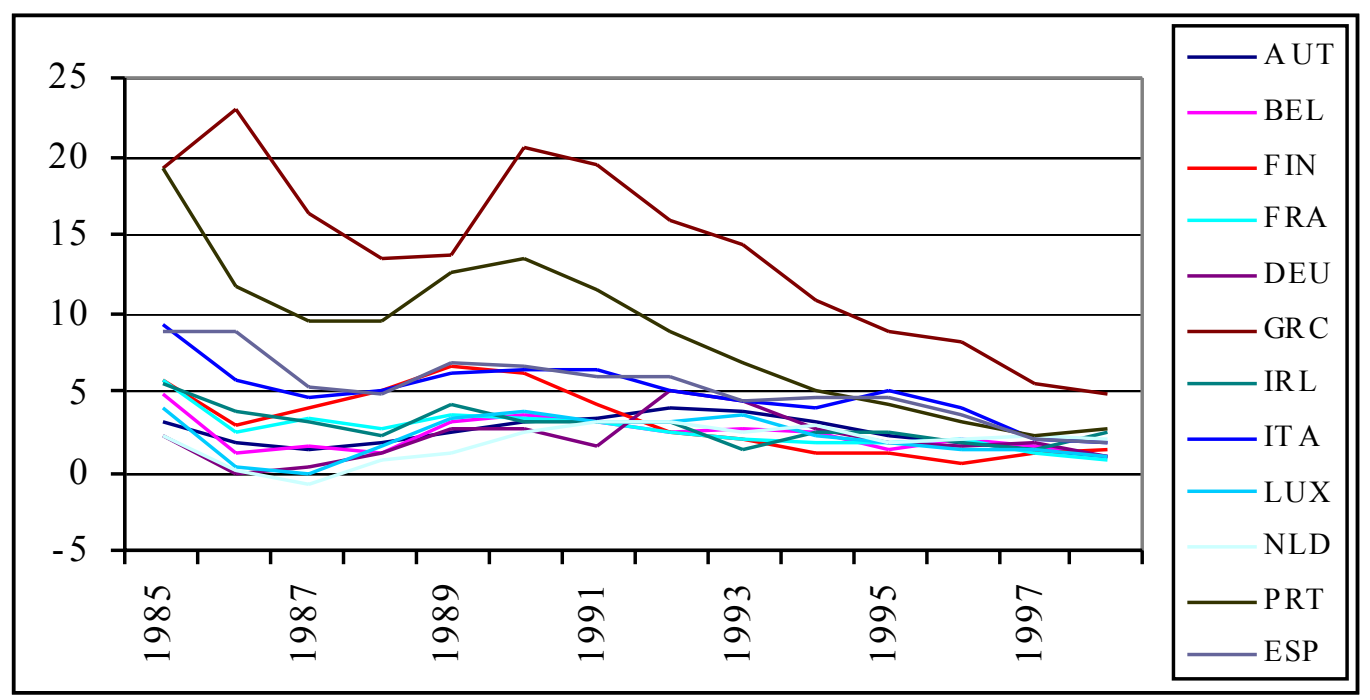

Fuente: elaboración propia con datos del Banco Mundial.

En el Gráfico 2 se representa en el eje vertical izquierdo la evolución de las tasas de crecimiento en trece estados miembros de la UE, en relación a la tasa de crecimiento de la UE para el periodo 19772000. Las divisiones verticales representan los distintos estadios de integración económica en la UE. El periodo entre 1977 y 1986 marca el apogeo de la fase de Unión Aduanera. Las barras situadas entre 1986, el año de la firma del Acta Única Europea, y 1993, el año de entrada en vigor del Mercado Único, delimitan la fase de transición hasta el Mercado Único. El periodo entre 1993 y el año 2000 cubre la fase de libre circulación de los factores económicos en el interior del Mercado Único.

En la mayoría de los casos se observa un progresivo solapamiento entre las tasas de crecimiento nacionales y las tasas de crecimiento de la UE (Aghion \& Saint-Paul, 1998). Este solapamiento es particularmente evidente en la mayoría de aquellos países que tradicionalmente han formado el núcleo duro de la economía de la UE: Alemania, Francia, Bélgica e Italia. La única excepción en este sentido está representada por los Países Bajos, que crecen por encima de la media de la UE a partir de la puesta en marcha del Mercado Único ${ }^{2}$ (Gráfico 2).
Pero no son sólo los países del núcleo duro los que adecuan sus pautas de crecimiento económico a las pautas de crecimiento de la UE. Una convergencia en crecimiento más acusada -si cabe- se observa en países que se han incorporado a las CCEE durante el período de análisis. Este es el caso de España o Portugal, que pasan de tener ciclos económicos relativamente distintos a los del resto de los países miembros de la UE, con anterioridad a su incorporación a las CCEE, a tasas de crecimiento que se acercan con rapidez a las de los restantes países miembros (B arrios \& De Lucio, 2002). Evoluciones similares se aprecian en los tres países que se incorporaron a la UE en 1995. Mientras que a finales de los años ' $70 \mathrm{y}$ principios de los ' 80 las tasas de crecimiento en Austria, Finlandia y Suecia seguían cánones muy distintos a los de los otros miembros de la UE, desde mediados de los años ' 80 -y en particular desde su incorporación a la UE- sus tasas de crecimiento se acercan cada vez más a las europeas. El caso más espectacular es el de Austria, cuyos niveles de crecimien-

${ }^{2}$ En el caso alemán el proceso de reunificación económica y la asimilación de la antigua República Democrática Alemana supuso un paréntesis en el proceso de adecuación del ciclo económico alemán al europeo durante los años que siguieron a la reunificación. A partir de 1995, la adecuación de ambos ciclos ha vuelto a ser prácticamente total. 
to desde 1995 son casi los mismos que la media de la Unión.

Las únicas excepciones a la regla las constituyen dos de los tres países que menos se han involucrado en el proceso de integración económica. El Reino Unido y Dinamarca no sólo han decidido no participar en la moneda única, sino que también son los países que mayores reticencias han mostrado durante todo el proceso de integración. No es de extrañar que una de las posibles consecuencias de estas reticencias sea la inadecuación de sus ciclos económicos nacionales a los ciclos económicos de la UE. Esta inadecuación es más marcada en el caso del Reino Unido que en el de Dinamarca. El ciclo económico del Reino Unido, muy ligado al de Estados Unidos, parece ir ligeramente por delante del europeo, ya que tanto sus fases expansivas como las recesivas se suelen producir un par de años antes que en el resto de Europa (Barrios et al., 2002). También los dientes de sierra de la evolución del crecimiento son más acusados en el Reino Unido que en el resto de la UE. En el caso de Dinamarca el mayor desajuste se produjo en la fase de transición al Mercado Único. Y aunque recientemente ha ha- bido un acercamiento, el ciclo económico danés sigue siendo sustancialmente diferente al del resto de la UE (Gráfico 2).

El acercamiento de los ciclos nacionales al europeo es refrendado cuando se analizan las correlaciones entre las tasas de crecimiento nacional y las tasas de crecimiento europeo en los diferentes estadios de integración (Cuadro 1). En la mayoría de los casos, los coeficientes de correlación son altamente positivos y significativos, y por regla general, el grado de correlación aumenta a medida que se avanza el proceso de integración.

Las únicas excepciones son las anteriormente mencionadas del Reino Unido, Dinamarca y Luxemburgo. Irlanda, a pesar de tener un ciclo económico todavía relativamente alejado de la UE y cercano al del Reino Unido, tiene una evolución contraria a este último. Mientras que las tasas de crecimiento del Reino Unido -que no ha adoptado el Euro- tienden a alejarse de las tasas de crecimiento de la UE desde 1993, las tasas de crecimiento de Irlanda, que sí es miembro de la zona Euro, se acercan cada vez más a las del resto de la UE (Guadro 1).

Gráfico 2. Evolución de las tasas de crecimiento nacional con respecto a la Europea (eje izquierdo) y evolución de la convergencia sigma en el interior de cada Estado (eje derecho).
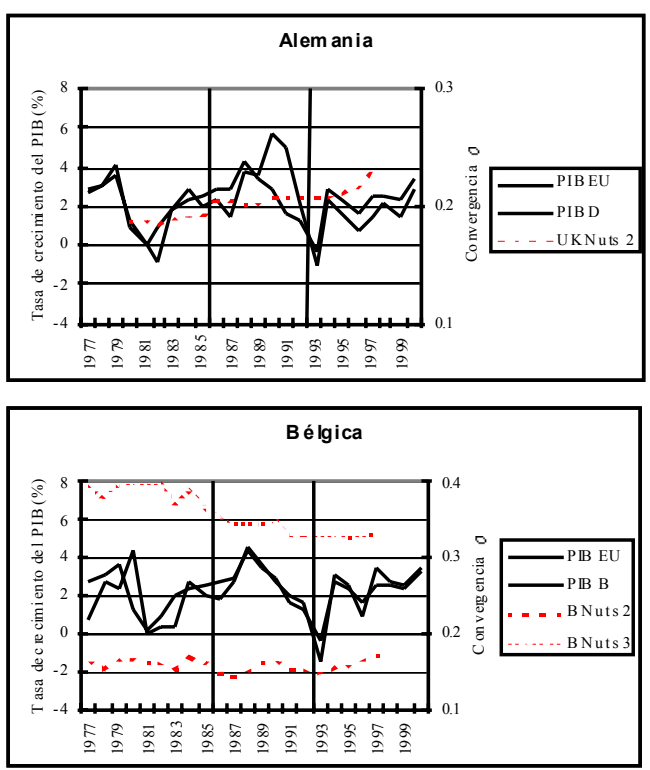
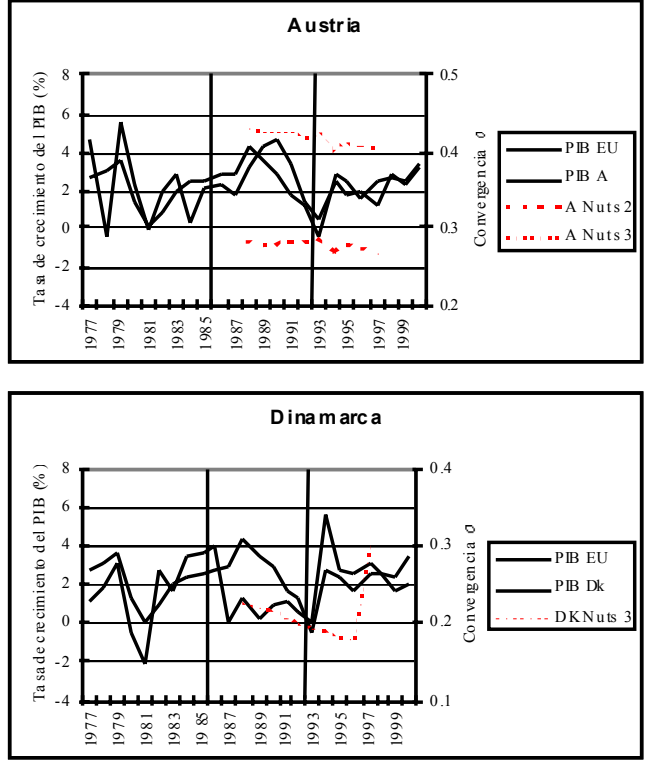
Integración económica y desequilibrios territoriales en la Unión Europea
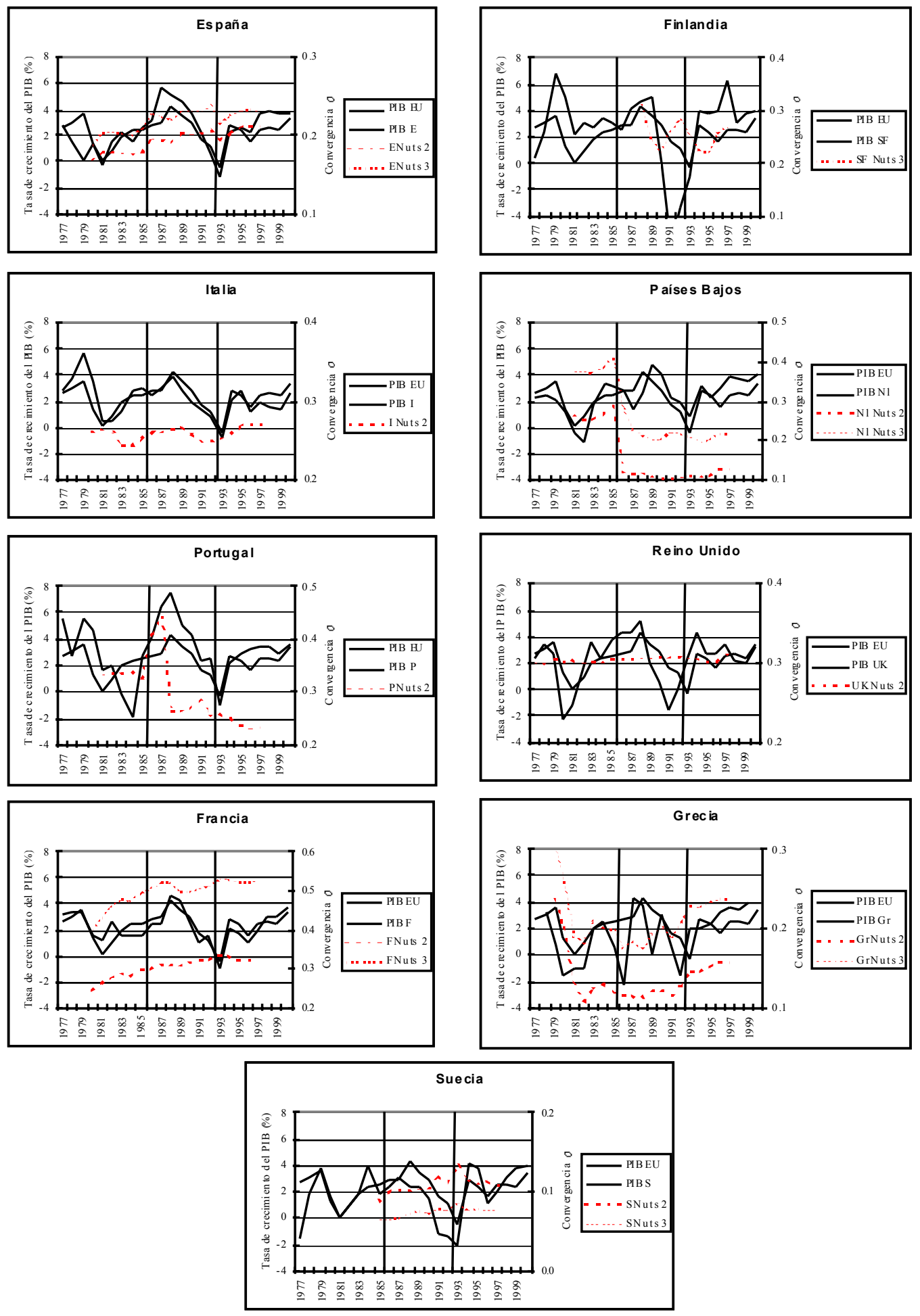

Fuente: elaboración propia a partir de datos EUROSTAT. 
La convergencia en los ciclos de crecimiento de los estados europeos contrasta fuer temente con la evolución de las pautas de crecimiento en otras áreas del mundo. El proceso de globalización económica actual no ha sido suficiente para producir un acercamiento en los ciclos económicos entre los tres principales centros económicos mundiales. El coeficiente de correlación entre Estados Unidos y la UE ha permanecido relativamente estable en los tres estadios del periodo. Se trata de una relación positiva, pero no especialmente fuerte ni significativa.

El caso de Japón es incluso más revelador, ya que a pesar de partir de un ciclo económico relativamente similar al europeo durante gran parte de los años ' 80 , la crisis económica japonesa ha hecho que durante los '90 los ciclos de ambos espacios hayan div ergido claramente (Cuadro 1).

\section{Integración económica y polarización regional}

Sin embargo, la convergencia económica que parece presidir el proceso de integración europea en casi todos los ámbitos no es universal. En el campo de los desequilibrios territoriales el proceso de integración económica no está siendo acompañado de convergencia (López-Bazo et al., 1999; Magrini, 1999; Cheshire \& Magrini, 2000; Boldrin \& Canova, 2001; Gil Canaleta et al. 2002).

A primera vista la ausencia de convergencia es difícil de apreciar. El rápido crecimiento, en los últimos años, de algunos de los estados europeos que tenían niveles de PIB per cápita claramente por debajo de la media europea es la base de los postulados neoclásicos y neo-ricardianos de la existencia de un proceso de convergencia territorial (Barro \& Sala-i-Martín, 1991; Sala-i-Martín, 1996). El hecho de que la reducción de las disparidades entre estados se haya producido a partir de la firma del Acta Única (que coincide con la entrada de España y Por tugal), y en el caso de I rlanda, a partir de principios de los años ' 90 (Gráfico 3), ha llevado incluso a algunos (por ejemplo, Comisión de las Comunidades Europeas [1995] o Leonardi [1995]) a establecer -de manera algo simplista, desde nuestro punto de vistavínculos directos entre el proceso de integración, los esfuerzos de la UE por reducir las disparidades regionales y alcanzar una mayor cohesión socioeconómica y la reducción de las disparidades entre el centro y la periferia.

La tendencia a la convergencia entre regiones queda refrendada si se analizan las tasas de crecimiento regional con respecto al PIB inicial de cada espacio. Esto es lo que se hace en el Gráfico 4, en el que se presentan las tasas de crecimiento regional para cada una de las regiones europeas (en el eje de ordenadas) y el PIB inicial (en el eje de abscisas). Cada uno de los subgráficos representa un estadio en el proceso de integración europea, de acuerdo con la división temporal establecida en el apartado anterior.

Los resultados de este tipo de análisis se asemejan bastante a los de los economistas neoclásicos (Barro \& Sala-i-Martín, 1991; B arro, 1991; Salai-Martín, 1996). En todos los gráficos se aprecia una ligera convergencia regional independientemente del estadio de integración económica en el que nos encontremos. A la vista de estos resultados, el impacto de la integración económica llevada a cabo en las dos últimas décadas sobre los desequilibrios regionales se podría considerar irrelevante, ya que no alteraría la tendencia general a la convergencia. Las distintas fases del proceso de integración dejan la función de regresión prácticamente inalterada. Se da convergencia regional en todos los periodos, aunque el nivel de convergencia regional es bajo.

Además, las tasas de convergencia de este análisis son similares a las obtenidas por la mayoría de los estudios sobre convergencia (Armstrong, 1995; Marcet \& Canova, 1995; De la Fuente, 1997; López-Bazo et al., 1999; Cuadrado et al., 1999). Se trata de tasas de convergencia que oscilan entre el 0,5 y el $2 \%$ anual defendido por las teorías neoclásicas.

No obstante, numerosos estudios (Quah, 1996; Magrini, 1999; Rodríguez-Pose, 1999) han demostrado que los resultados de este tipo de análisis están afectados por un serio problema de autocorrelación espacial, que unido al problema de la falacia de Galton o regresión hacia la media descrito por Quah, tienden a aumentar los coeficientes de convergencia de aquellas áreas cuyas econo- 
Cuadro 1. Correlaciones entre las tasas de crecimiento nacional y europea en los distintos estadios de integración económica.

\begin{tabular}{|c|c|c|c|c|}
\hline & Unión Aduanera & Transición & Mercado Unico & Total \\
\hline & $1977-1986$ & $1986-1993$ & $1993-2000$ & $1977-2000$ \\
\hline \multirow[t]{2}{*}{ Alemania } & $0,928^{* *}$ & 0,083 & $0,979^{* *}$ & $0,707^{* *}$ \\
\hline & 0,000 & 0,860 & 0,000 & 0,000 \\
\hline \multirow[t]{2}{*}{ Austria } & 0,459 & 0,443 & $0,803^{*}$ & $0,533^{* *}$ \\
\hline & 0,214 & 0,320 & 0,017 & 0,007 \\
\hline \multirow[t]{2}{*}{ Bélgica } & 0,375 & $0,895^{* *}$ & $0,973^{* *}$ & $0,732^{* *}$ \\
\hline & 0,320 & 0,006 & 0,000 & 0,000 \\
\hline \multirow[t]{2}{*}{ Dinamarca } & 0,664 & 0,026 & 0,542 & 0,399 \\
\hline & 0,051 & 0,956 & 0,165 & 0,053 \\
\hline \multirow[t]{2}{*}{ España } & 0,247 & 0,872 & $0,905^{* *}$ & $0,702^{* *}$ \\
\hline & 0,522 & 0,010 & 0,002 & 0,000 \\
\hline \multirow[t]{2}{*}{ Finlandia } & 0,231 & $0,857^{*}$ & $0,871^{\text {** }}$ & $0,456^{*}$ \\
\hline & 0,549 & 0,014 & 0,005 & 0,025 \\
\hline \multirow[t]{2}{*}{ Francia } & 0,629 & $0,933^{* *}$ & $0,933^{* *}$ & $0,831^{* *}$ \\
\hline & 0,000 & 0,002 & 0,001 & 0,000 \\
\hline \multirow[t]{2}{*}{ Grecia } & $0,800^{* *}$ & 0,321 & $0,900^{* *}$ & $0,617^{* *}$ \\
\hline & 0,010 & 0,482 & 0,002 & 0,001 \\
\hline \multirow[t]{2}{*}{ Irlanda } & 0,388 & 0,378 & 0,683 & 0,322 \\
\hline & 0,303 & 0,403 & 0,062 & 0,113 \\
\hline \multirow[t]{2}{*}{ Italia } & $0,829^{* *}$ & $0,957^{* *}$ & $0,914^{* *}$ & $0,786^{* *}$ \\
\hline & 0,006 & 0,001 & 0,002 & 0,000 \\
\hline \multirow[t]{2}{*}{ Luxemburgo } & 0,621 & 0,518 & $-0,427$ & 0,321 \\
\hline & 0,074 & 0,234 & 0,291 & 0,126 \\
\hline \multirow[t]{2}{*}{ Países B ajos } & $0,808^{* *}$ & 0,392 & $0,891^{\text {** }}$ & $0,676^{* *}$ \\
\hline & 0,008 & 0,384 & 0,003 & 0,000 \\
\hline \multirow[t]{2}{*}{ Portugal } & 0,300 & $0,900^{* *}$ & $0,875^{* *}$ & $0,634^{* *}$ \\
\hline & 0,433 & 0,006 & 0,004 & 0,001 \\
\hline \multirow[t]{2}{*}{ Reino Unido } & $0,697^{*}$ & 0,752 & 0,447 & $0,576^{* *}$ \\
\hline & 0,037 & 0,051 & 0,266 & 0,003 \\
\hline \multirow[t]{2}{*}{ Suecia } & 0,406 & $0,835^{*}$ & $0,942^{* *}$ & $0,633^{* *}$ \\
\hline & 0,279 & 0,019 & 0,000 & 0,001 \\
\hline \multirow[t]{2}{*}{ Estados Unidos } & 0,581 & 0,567 & 0,547 & $0,430^{*}$ \\
\hline & 0,101 & 0,184 & 0,137 & 0,036 \\
\hline \multirow[t]{2}{*}{ Japón } & $0,784^{*}$ & $0,856^{*}$ & $-0,118$ & 0,339 \\
\hline & 0,012 & 0,014 & 0,781 & 0,105 \\
\hline
\end{tabular}

Los símbolos ${ }^{* *} \mathrm{y}^{*}$ indican coeficientes de correlación significativos a niveles de 1 y $5 \%$ respectivamente.

Fuente: elaboración propia a partir de datos EUROSTAT.

mías están íntimamente ligadas. Por eso, resulta conveniente llevar a cabo un análisis de la evolución de las disparidades regionales en el interior de cada país para observar si la tendencia a la convergencia que se aprecia en el Gráfico 4 queda refrendada.

Los resultados de este tipo de análisis, sin embargo, presentan un panorama radicalmente diferente; en lugar de predominar la tendencia a la convergencia regional a medida que avanza el proceso de integración europea, la convergencia regional va dando paso a un proceso de divergencia en numerosos países europeos, tanto en Europea Occidental (Gil Canaleta et al., 2002; Puga, 2002) como en los países de Europa Central y Oriental
(Petrakos, 2001). Una primera indicación de este cambio surge del análisis de convergencia sigma para la regiones NUTS 2 y 3, incluido en el eje vertical derecho del Gráfico 2. Mientras que en muchos países se observa estabilidad o una ligera conv ergencia hasta principios de los años ' 90 , desde la puesta en marcha del Mer cado Único la tendencia se invierte. Este es el caso de Bélgica, Grecia, España, los Países Bajos, Dinamarca, Italia, el Reino Unido, Alemania y Finlandia, en el que las disparidades regionales se acentúan a medida que se profundiza en el proceso de integración económica europea. Las excepciones a la regla son Austria, Por tugal, Suecia y Francia. En el caso de Francia la evidencia es confusa, ya que, además de ex- 
Gráfico 3. Evolución del PIB per cápita (medido en PPS) con respecto a la media europea de los cuatro países beneficiarios de los fondos de cohesión entre 1977 y 2000.

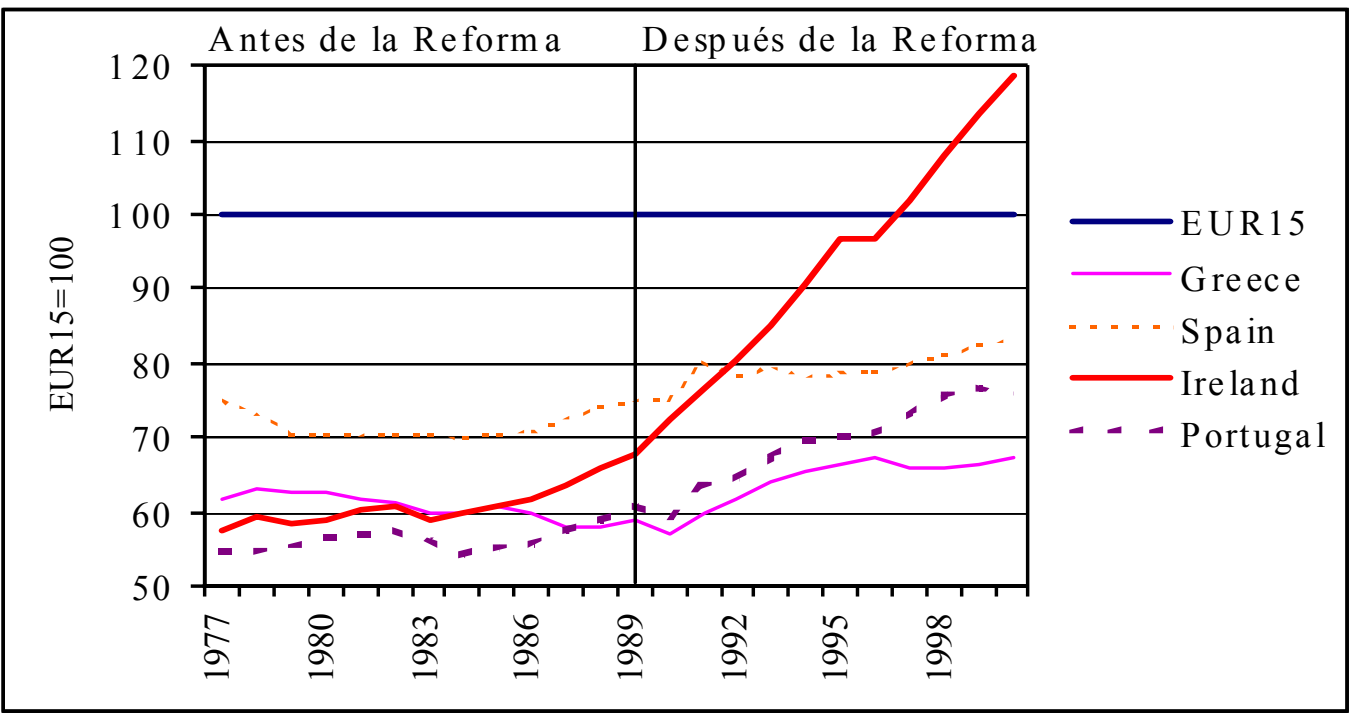

perimentar ya un proceso de divergencia en las fases anterior es del proceso de integración, desde la puesta en marcha del Mercado Único se observa una estabilidad a nivel NUTS 3, y una ligera convergencia a nivel NUTS 2.

Este proceso de mayor divergencia nacional a medida que se profundiza en la integración europea, se refrenda cuando se lleva a cabo el mismo análisis del Gráfico 4 a nivel nacional en aquellos países para los que se poseen series completas de datos (Gráfico 5).

Los resultados del análisis de convergencia presentados en el Gráfico 5 difieren en la mayoría de los casos de la convergencia presentada en el Gráfico 4. Se observa, en primer lugar, una transformación impor tante en las pautas de crecimiento a medida que se avanza en las fases de integración económica. A mayor nivel de integración, may or dinamismo de las áreas centrales de los estados. Las regiones que más se benefician del proceso de integración son aquellas que están más integradas en los circuitos económicos europeo y globales, mientras que aquellas de carácter semiperiférico o con peor es condiciones iniciales crecen menos. Se va pasando pues de un proceso de convergencia regional a nivel nacional a uno de divergencia, a medida que se queman etapas de integración económica (Gráfico 5). Esto ocurre en la gran mayoría de los países para los que se tienen series completas de datos. La pendiente de la línea de regresión pasa de ser negativa, en la fase de Unión Aduanera, a convertirse en una pendiente positiva ya en el Mercado Único. En aquellos países como Grecia, en los que la pendiente de la recta de regresión sigue siendo negativa, se observa una fuer te desaceleración del proceso de convergencia (Gráfico 5). La excepción a la regla es Francia, cuya convergencia es debida al escaso dinamismo de la región parisina durante el periodo del Mercado Único.

La tendencia a la divergencia a medida que se avanza en el proceso de integración económica europea corrobora la hipótesis de convergencia a nivel europeo, pero divergencia a nivel nacional (Rodríguez-Pose, 1998; López-Bazo et al., 1999; Puga, 2002). Por consiguiente, se podría afirmar que la evolución de las disparidades territoriales durante el proceso de integración europea estaría más cercana a los postulados de las teorías de crecimiento endógeno o a la nueva geografía económica que a las teorías neoclásicas. Frente a la convergencia que se da en muchos otros apartados, la integración económica parece que a mediano y largo plazo está beneficiando -y seguramente va a beneficiar- a aquellos espacios que gozan ya de 
Gráfico 4. Relación entre el PIB per cápita regional inicial (medido en pps) y el crecimiento del mismo en cada uno de los estadios de integración económica europea.
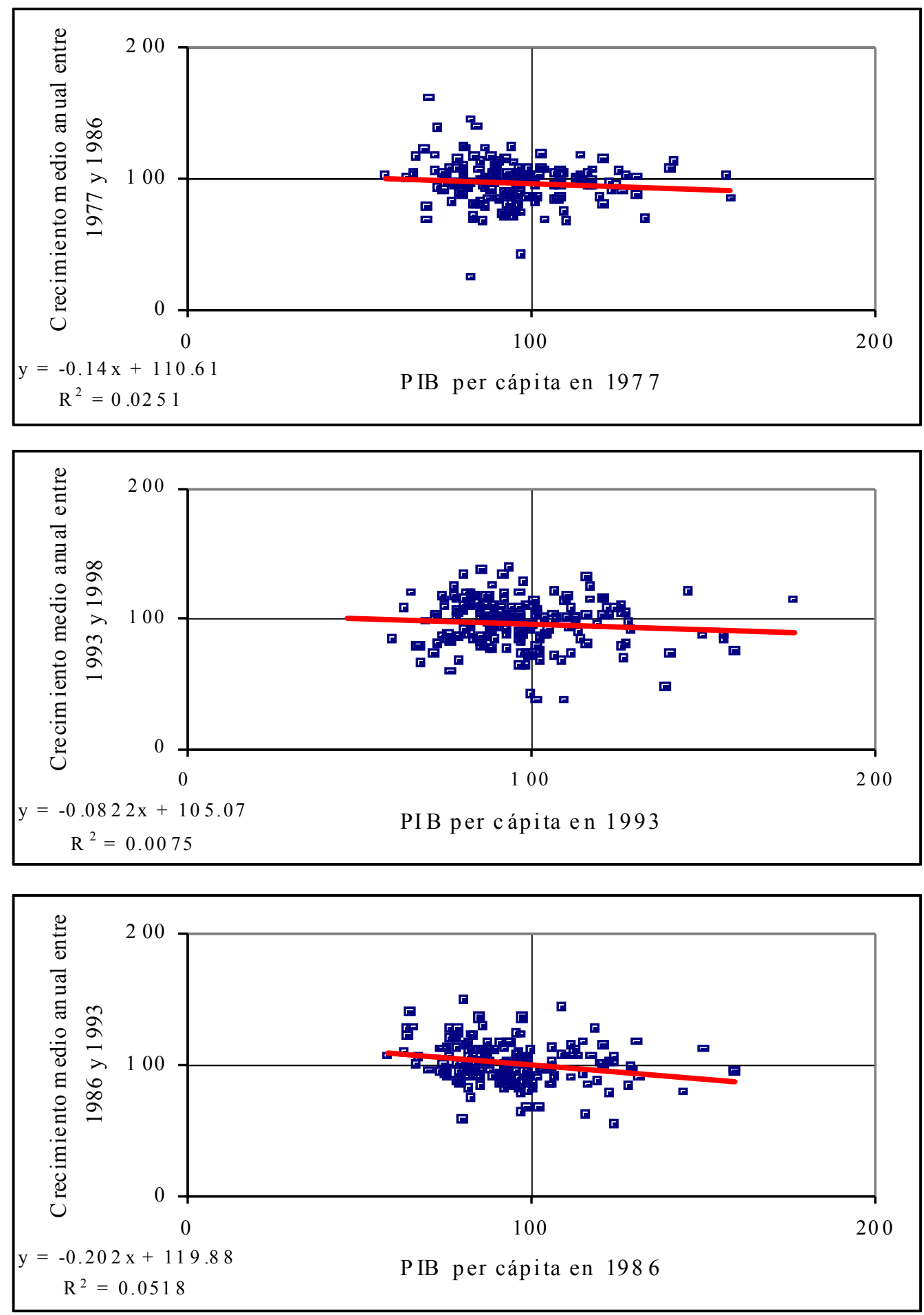

Fuente: elaboración propia a partir de datos EUROSTAT. 

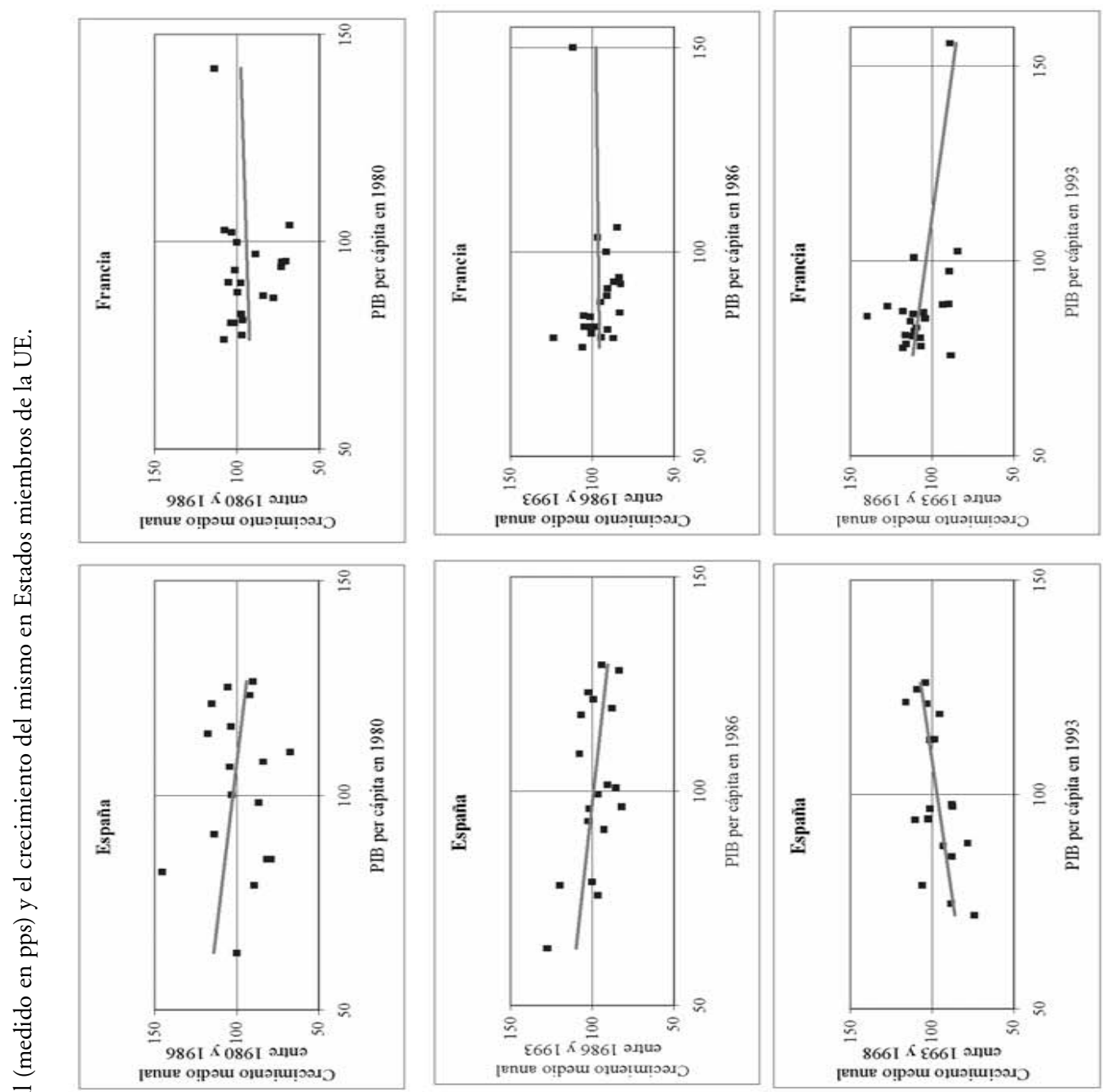

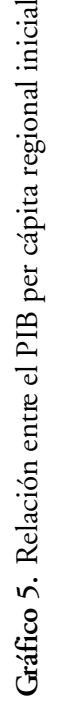
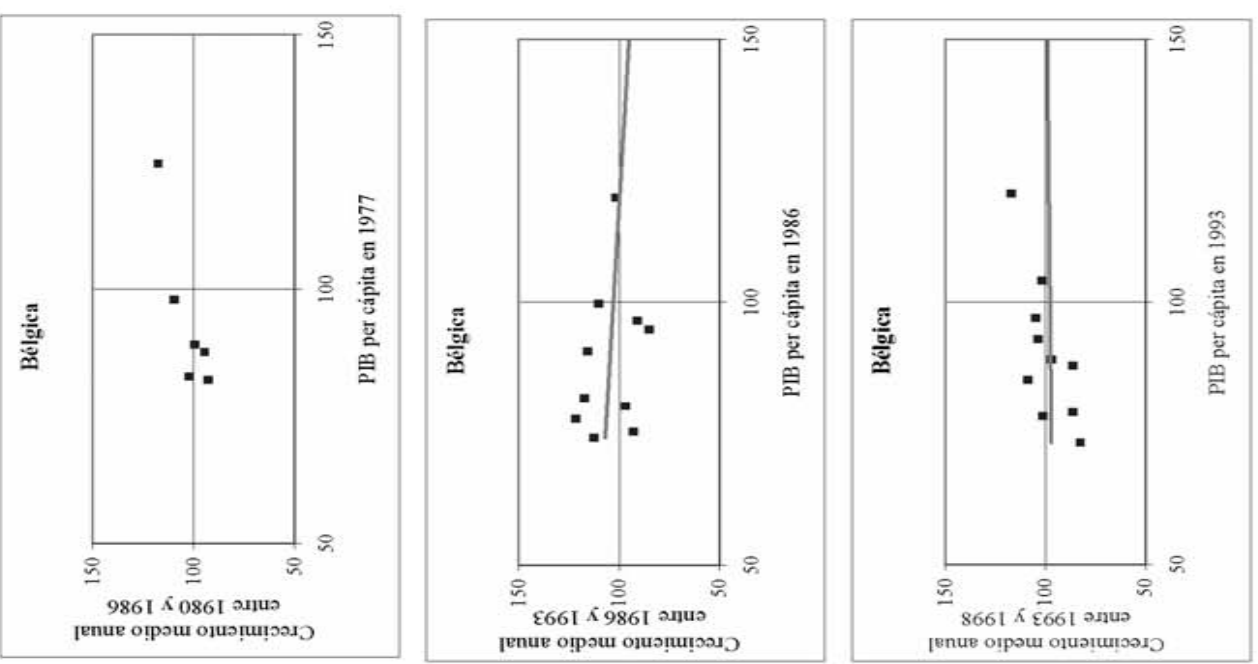

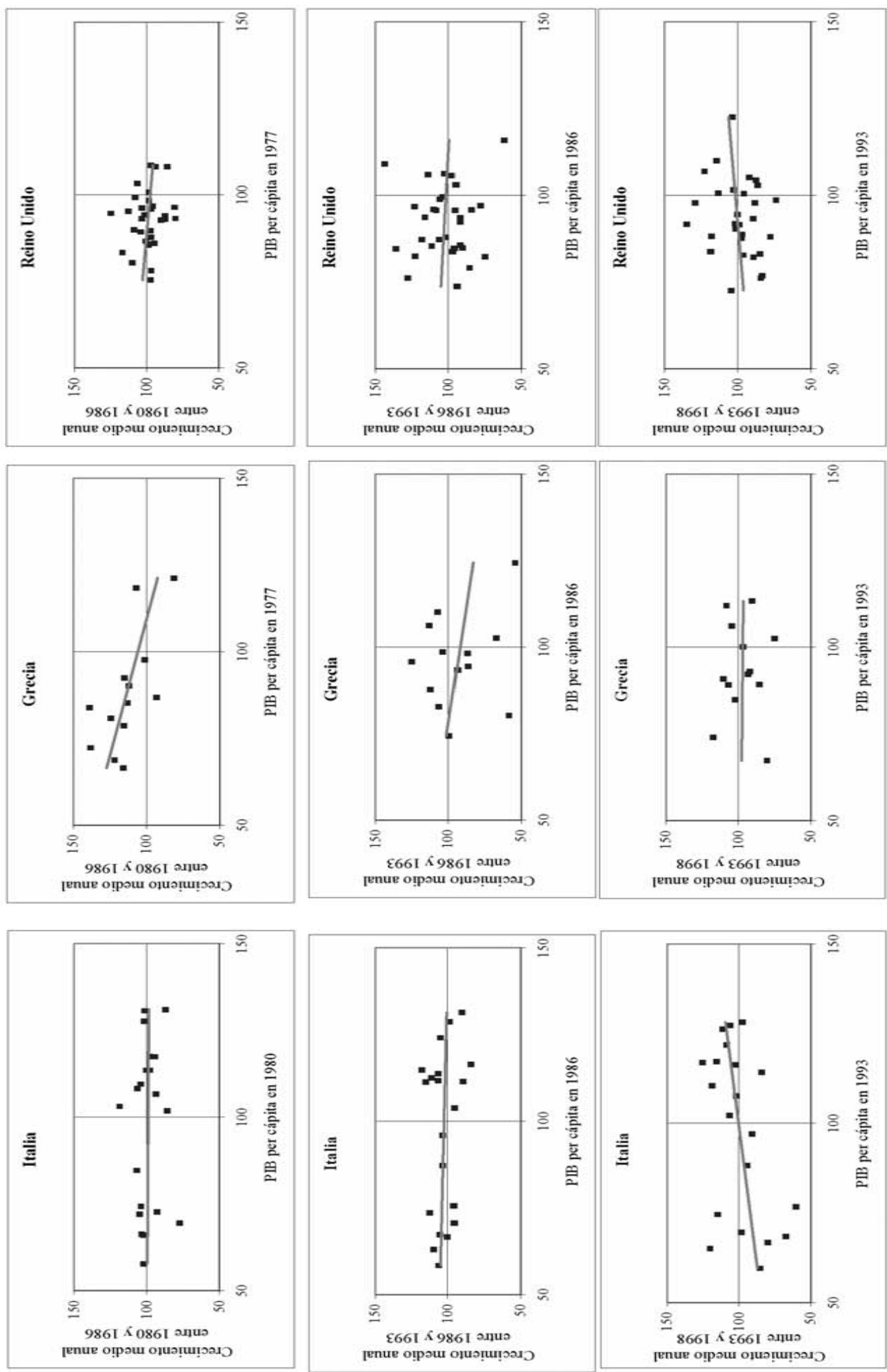

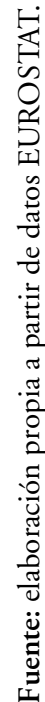


ventajas comparativas y de economías de aglomeración, mientras que los espacios más atrasados, a pesar del esfuerzo de las políticas europeas, están en peores condiciones para recopilar los beneficios del proceso de integración.

Los factores detrás de los procesos paralelos de convergencia a nivel europeo y divergencia en el seno de la gran mayoría de los estados merecen may or atención de la que, por motivos de espacio, se puede dedicar en este ar tículo. Ello no obstante, nos gustaría esbozar tres argumentos que pueden servir de base para una investigación del porqué de estos contrastes en la evolución de las disparidades entre estados y en el interior de cada Estado. En primer lugar, cabría destacar el todavía imperfecto grado de integración a nivel supranacional. A pesar de que con la implantación del Mercado Único el 1 de enero de 1993 se abrier on las fronteras a la libre circulación de bienes, servicios, capital y mano de obra, y con la entrada en vigor del Euro se ha eliminado otra barrera para la integración, la movilidad de los factores productivos entre países -en especial en lo referente a la mano de obra y los servicios- es todavía relativamente limitada (Baldwin \& Wyplosz, 2003). Las diferencias persistentes en los sistemas institucionales y jurídicos y las disparidades políticas y culturales siguen representando importantes barreras para la movilidad de los factores. Estas barreras son, en cambio, mucho menos impor tantes en el interior de cada Estado, por lo que una mayor movilidad intraestatal puede estar favoreciendo el desarrollo de las economías de aglomeración.

Un segundo factor que puede estar detrás de la dicotomía convergencia-divergencia encontrada está ligado al tipo de políticas enfrentadas desarrolladas a nivel europeo y nivel nacional. Mientras que el objetivo de las políticas de desarrollo europeo -fondos estructurales y fondos de cohesión- es el promover el desarrollo en aquellos países y áreas más atrasadas de la UE (Boldrin \& Canova, 2001), muchas de las políticas estatales tienen como objetivo fundamental promover el crecimiento nacional, sin prestar atención a los problemas de cohesión en el interior de cada Estado (Midelfart-Knarvik \& Overman, 2002). Dicha combinación puede, pues, favor ecer el creci- miento en los países de la periferia, pero no necesariamente en las regiones más pobres de dichos países, sino en sus espacios más dinámicos.

Finalmente, este efecto se vería a su vez reforzado por la propia estructura de los fondos de desarrollo europeos. Aproximadamente el 50\% de los fondos europeos destinados al desarr ollo de las áreas más atrasadas de la Unión se dedican a la construcción de infraestructuras. La mayoría se utiliza para la construcción de infraestructuras de transporte, estructuradas en la may oría de los casos en redes suprarregionales e incluso transeuropeas. Esta situación, más que favorecer el desarrollo de las áreas más periféricas, puede estar contribuyendo al desarrollo de aquellos principales nodos de transporte (como por ejemplo, Madrid, Lisboa, Roma, Atenas o Dublín), donde se asientan las empresas más competitivas y la mano de obra más cualificada, promoviendo de nuevo el crecimiento nacional, pero no especialmente el de las áreas más pobres del país (Rodríguez-Pose \& Fratesi, 2004).

\section{Conclusiones}

La integración económica europea parece estar cada vez más asociada a un proceso de divergencia económica entre regiones. Si bien es cierto que las economías europeas se asemejan cada vez más, y que los mismos factores condicionan su funcionamiento, desde un punto de vista territorial las disparidades económicas en el interior de cada país están aumentando a medida que se queman etapas de integración. Frente a un proceso de convergencia entre estados, y a unos espacios centrales cada vez más incorporados a la economía europea y mundial, se alzan muchos espacios periféricos cuya capacidad para competir en una economía más integrada y globalizada es escasa. Es todavía relativamente pronto para tener una visión definitiva de cuál ha sido el impacto del proceso de integración económica, pero los primeros resultados ponen de manifiesto los problemas que muchas regiones europeas van a tener para competir con las áreas centrales. Además, es probable que si se mantienen las tendencias actuales, la convergencia que todavía se observa entre países disminuya y se convierta, al igual que ocurre en el seno de la mayoría de los estados europeos, 
en divergencia. Bajo estas condiciones, se antoja cada vez más difícil que el objetivo europeo de cohesión económica y social pueda alcanzarse en un futuro relativamente próximo.

\section{Referencias bibliográficas}

Aghion, Ph. \& G. Saint-Paul (1998). “Uncovering some causal relationships between productivity growth and the structure of economic fluctuations: a tentative survey". Labour, 12, 2: 279-303.

Archibugi, D. \& S. Iammarino (2002). "The globalization of technological innovation: definition and evidence". Review of Intemational Political Economy, 9, 1: 98-122.

Armstrong, H. W. (1995). "Convergence among regions of the European Union. 1950-1990". Papers in Regional Science, 74: 143-152.

Audretsch, D. B. \& M. P. Feldman (1996). "R\&D spillovers and the geography of innovation and production". American Economic Review, 86: 630-40.

Barrios, S. \& J. J. de Lucio (2002). "Economic integration and Regional Business Cycles: evidence from the Iberian regions". CORE discussion paper 2002/73.

Barrios, S., M. Brülhart, R. J. Elliot y M. Sensier (2002). "A tale of two cycles: co-fluctuations between UK regions and Europe". CORE working paper.

Barro, R. J. (1991). "Economic growth in a crosssection of countries". Quarterly Journal of Economics, 10: 407-443.

Barro, R. J. \&X. Sala-i-Martín (1991). "Convergence across states and regions". Brookings Papers on Economic Activity, 1: 107-182.

Barry, F. (2003) "Economic integration and convergence processes in the EU cohesion countries". Journal of Common Market Studies, 41: 897-921.

Begg, I. (2003). "Complementing EMU: rethinking cohesion policy”. Oxford Review of Economic Policy, 19, 1: 161-179.

Boldrin, M. \& F. Canova (2001). "Inequality and convergence in E urope's r egions: reconsidering European regional policies". Economic Policy, 16: 207-253.

Cappelen, A., F. Castellacci, J. Fagerberg y B.
Verspagen (2003). “The impact of EU regional support on growth and convergence in the European Union". Journal of Common Market Studies, 41, 4: 621-644.

Cecchini, P. (1988). The European challenge 1992: the benefits of a single market. Aldershot : Wildwood House.

Cheshire, P. C. \& S. Magrini (2000). "Endogenous processes in European regional growth: convergence and policy". Growth and Change, 31: 455-479.

Cohen, W. M. \& D. A. Levinthal (1989). "Innovation and learning: the two faces of R\&D”. Economic Journal, 99: 569-596.

Comisión de las Comunidades Europeas (1995). Competitiveness and cohesion: trends in the regions. Sixth periodic report on the social and economic situation and development of the regions in the Community. Bruselas-Lux emburgo: OPOCE.

Cuadrado Roura, J. R. (1996) "Convergencia nominal y convergencia real. El verdadero reto para España”. Cuadrado Roura, J. R. \& T. Mancha (comps.), España frente a la Unión Económica y Monetaria. Madrid: Civitas.

(2001). "Regional convergence in the European Union: from hypothesis to the actual trends". Annals of Regional Science, 35: 333-356.

Cuadrado-Roura, J. R., B. García-Greciano y J. L. Raymond (1999). "Regional convergence in productivity and productive structure: the Spanish case". Intermational Regional Science Review, 22: 35-53.

De la Dehesa, G. (1998). El reto de la unión económica y monetaria. La Coruña: Instituto de Estudios Económicos de Galicia Pedro Barrié de la Maza.

De la Fuente, A. (1997). "The empirics of growth and convergence: a selective review". Journal of Economic Dynamics and Control, 21: 23-73.

Demertzis, M. \& A. Hughes Hallet (1996). "Regional inequalities and the business cycle: and explanation of the rise in European unemployment". Regional Studies, 30, 1: 15-29.

Dosi, G. (1988). "Sources, procedures, and microeconomic effects of innovation". Journal of Economic Literature, 26: 1120-1171.

Fujita, M., P. Krugman y A. J. Venables (1999). The spatial economy: cities, regions and 
international trade. Cambridge, Mass.: MIT Press.

Gil Canaleta, C., P. Pascual Arzoz y M. Rapún Gárate (2002). "Structural change, infrastructure, and convergence in the regions of the European Union”. European Urban and Regional Studies, 9: 115-135.

Hanson, G. H. (1998). "Regional adjustment to trade liberalization". Regional Science and Urban Economics, 28: 419-44.

Hess, G. D. \& K. Shin (1997). "International and intranational business cycles". Oxford Review of Economic Policy, 13, 3: 93-109.

Leonardi, R. (1995). Convergence, cohesion and integration in the European Union. Basingstoke: Macmillan Press.

López-Bazo, E., E. Vayá, A. J. Mora y J. Suriñach (1999). "Regional economic dynamics and convergence in the European Union". Annals of Regional Science, 33, 343-70.

Lucas, R. E. Jr. (1988). "On the mechanics of economic development". Journal of Monetary Economics, 22: 3-42.

Magrini, S. (1999). "The evolution of income disparities among the regions of the European Union". Regional Science and Urban Economics, 29: 257-281.

(2003). "Regional

convergence". Dipartimento di Scienze Economiche. Università Ca’ Foscari di Venezia (mimeo).

Marcet, A. \& F. Canova (1995). "The poor stay poor: non-convergence across countries and regions”. Discussion paper 1265, Centre for Economic Policy Research.

Martin, R. (1997). "Regional unemployment disparities and their dynamics". Regional Studies 31, 3: 237-252.

Midelfart-Knarvik, K. H. \& H. G. Overman (2002). "Delocation and European integration: is structural spending justified?" Economic Policy, 17: 322-59.

Ottaviano, G. I.P. \& D. Puga (1998). "Agglomeration in the global economy: a survey of the "New Economic Geography". World Economy, 21: 707-31.

Overman, H. G. \& D. Puga (2003). "Unemployment clusters across Europe's regions and countries". Economic Policy, 17:
115-147.

Pack, H. (1994). "Endogenous growth theory. Intellectual appeal and empirical shortcomings". Journal of Economic Perspectives, 8: 55-72.

Petrakos, G. (2001) "Patterns of regional inequality in transition economies". European Planning Studies, 9: 359-383.

Petrakos, G., A. Rodríguez-Pose y A. Rovolis (2003). "Growth, integration and regional inequality in Europe". Research Papers in Environmental and Spatial Analysis, London School of Economics.

Puga, D. (1999). "The rise and fall of regional inequalities". European Economic Review, 43: 303-334.

(2002). "European regional policy in light of recent location theories". Journal of Economic Geography, 2: 373-406.

Quah, D. (1996). "Empirics for economic growth and convergence". European Economic Review, 40: 1353-1375.

Rodríguez-Pose, A. (1998) Dynamics of regional growth in Europe. Social and political factors. Oxford: Clarendon Press.

(1999). "Convergence or divergence? Types of regional responses to socio-economic change in Western Europe". Tijdschrift voor Economische en Sociale Geografie, 90: 363-378.

(2001). "Is R\&D investment in lagging areas of Europe worthwhile? Theory and empirical evidence". Papers in Regional Science, 80, 3: 275-95.

Rodríguez-Pose, A. \& U. Fratesi (2004). "Between development and social policies: the impact of European Structural Funds in Objective 1 regions". Regional Studies, 38, 1: 97-113.

Romer, P. M. (1986). "Increasing returns and long-run growth". Journal of Political Economy, 94: 1002-1037.

Sala-i-Martín, X. (1996). "Regional cohesion. Evidence and theories of regional growth and convergence". European Economic Review, 40: 1325-1352.

Storper, M. (1997). The regional world. Nueva York: Guilford Press.

Tondl, G. (2002). Convergence after divergence? Regional growth in Europe. Berlín: Springer. 